\title{
Análisis de los esfuerzos y fractura en el ánima de un cañón de 3"/ 50 calibre
}

\author{
A.L. Herrera-May ${ }^{(1)}$, L.A. Aguilera-Cortés ${ }^{(2)}$, J. Hernández-Hernández ${ }^{(3)}$ y P.J. García-Ramírez ${ }^{(1)}$ \\ Centro de Investigación en M icro y Nanotecnología de la Universidad Veracruzana (1), FIM EE-Universidad de \\ Guanajuato ${ }^{(2)}$,Escuela de Ingenieros de la Armada de M éxico, Veracruz ${ }^{(3)}$. \\ E-mails: leherrera@uv.mx, aguilera@ salamanca.ugto.mx y jagarcia@uv.mx
}

(Recibido: octubre de 2005; aceptado: febrero de 2006)

\begin{abstract}
Resumen
En este trabajo se presentan los análisis de esfuerzos y de fractura en el ánima de un cañón de 3"/50 calibre, sometido a presión interna de 95 MPa y con dos tipos de grietas utilizando los criterios de falla de la mecánica de la fractura linealmente elástica. En todos los casos se demostró que se podían aplicar estos criterios. Para el estudio se proponen modelos geométricos en 2-D y 3-D, considerando el rayado interno del ánima, mediante el método de elemento finito (MEF) y con la ayuda de un soft ware comercial. La presencia de grietas en el modelo 2-D, registró un incremento significativo del $68.35 \%$ en los esfuerzos de Von Mises, pero sin superar el esfuerzo de fluencia del acero ASTM A723. Se determinaron los factores de intensidad de esfuerzos (FIEs) en las grietas de los modelos geométricos y se realizó un estudio de la zona plástica. Los modelos geométricos propuestos del ánima de un cañón reducen el tiempo de cómputo y son de fácil ejecución para la obtención de una aproximación aceptable de los esfuerzos y el FIE que le ocasionan grietas internas.
\end{abstract}

Descriptores: Fractura, grietas, factor de intensidad de esfuerzos, esfuerzos, ánima cañón, método de elemento finito. PACS: 02.70.Dh; 46.50.+a; 81.40.Np

\begin{abstract}
Thefractureand stress anal y sis in thebore of can non 3"/50 cal i ber with in ter nal pres sure (95 M Pa) and two type of cracks us ing thefault cri te ria of lin early elas tic frac tureme chan ics arepresented. In all the cases wedem on strated that thesecri te ria could beap plied. $G$ eo met ricmod els in 2-D and 3-D were proposed con sider ing ther fled borewith fi niteel ement method (FEM) and com mer cial soft ware. Thepres ence of cracks in themodel 2-D reg is tered a sig nifi cant in crease of the $68.35 \%$ in Von M ises stress, but with out ex ceed theyield stress sted ASTM A723. Wefind stress in ten sity factors (SIFs) in the cracks of the geomet ric mod els and wemade a study of the plas ticzone. Thegeo met ricmod els pro posed of borecan non re ducetime of com pu ta tion and are of easy ex ecu tion for theob tain ing of an accept ableapproach of stress and the SIF that cause in ter nal cracks to it.
\end{abstract}

Keywords: Fracture, cracks, stressin ten sity factor, stress, boreof can on, fi niteel ement method. PA CS: 02.70.Dh; 46.50.+ a; 81.40.Np

\section{Introducción}

Las condiciones de operación severas son comunes en el ánima de un cañón en donde el desgaste de los materiales y la formación de grietas ocasionan altos gradientes de esfuerzos. La se- guridad del ánima es de vital importancia, ya que su operación con grietas en condiciones críticas podrían ocasionar accidentes catastróficos, por lo que es muy importante conocer los esfuerzos y la distribución de éstos en el ánima del cañón, en especial, en el inicio del rayado, donde el cañón 
está sometido a las condiciones más severas de operación (Bache, 1996). Dado que los análisis de fractura en cañones son muy confidenciales, y por ende, de difícil acceso, es necesario realizar estudios analíticos y experimentales de varios tipos de grietas con el objetivo de conocer el grado de peligrosidad de éstas en los cañones. Uno de los investigadores que ha realizado estudios de grietas en cañones de la Armada de USA es Underwood et al . (1998);(2001).

Un parámetro que establece el índice de severidad de una grieta es el factor de intensidad de esfuerzos que al utilizarse en los criterios de falla de la mecánica de la fractura linealmente elástica (González, 1998) indican la gravedad de ésta. Levy et al. (2003), han realizado estudios del factor de intensidad de esfuerzos en cilindros erosionados sometidos a presión interna.

Debido al interés del personal de la Escuela de Ingenieros de la Armada de México en conocer los esfuerzos y la peligrosidad de ciertos tipos de grietas en el ánima de un cañón de 3"/50 calibre, se presenta el siguiente trabajo en donde se realizaron los análisis de esfuerzos y de fractura para este modelo de cañón, considerando cierto tipo de grietas en el inicio del rayado, las cuales fueron sometidas a una presión interna de $95 \mathrm{MPa}$. En este tipo de cañones las municiones son del tipo fijo, diseñadas para desarrollar una velocidad inicial de $822.96 \mathrm{~m} / \mathrm{s}$, con un alcance máximo horizontal de 10,972.8 m y una altura máxima de $6400.8 \mathrm{~m}$ aproximadamente (Bache, 1996). En la figura 1 se muestra la ubicación del proyectil en el ánima de un cañón.

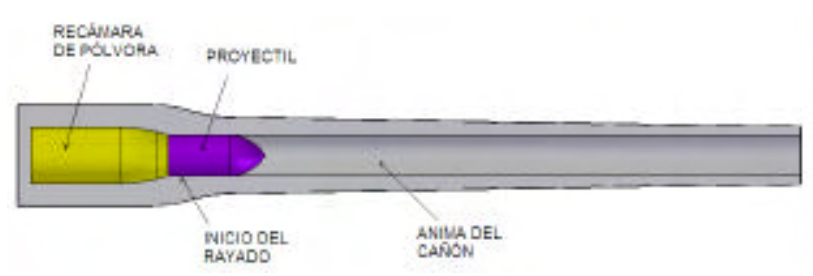

Figura 1. Proyectil en el ánima de un cañón

La mecánica de la fractura relaciona el tamaño, la geometría de una grieta y las fuerzas que originan la fractura de un componente de forma y dimensiones conocidas. Para esto, se apoya en el cálculo de la distribución de los esfuerzos, defor- maciones, desplazamientos alrededor de una grieta y en el establecimiento de los balances de energía que tienen lugar durante la extensión de una grieta.

\section{Criterios de falla}

En el análisis de fractura es necesario conocer las condiciones en las cuales el elemento que contiene una o varias grietas fallará, como son: la carga máxima que puede aplicarse, el número de ciclos necesarios para que la pieza falle por fatiga, la longitud máxima que puede alcanzar la grieta sin llegar a la fractura, etc. Una forma aproximada de conocer estas condiciones es mediante los siguientes criterios (Galaviz, 2003): esfuerzo tangencial máximo, máxima razón de energía liberada y mínima densidad de energía de deformación. En donde las dos últimas son las más utilizadas y tienen como fundamento el factor de intensidad de esfuerzos.

Considerando la teoría de Irwin se obtiene un valor crítico de la razón de energía liberada $G_{c}$ en donde la grieta comenzará a propagarse de acuerdo al modo de carga que se presente, como se indica a continuación:

$$
G_{c}=\left\{\begin{array}{cl}
\frac{K_{c}^{2}}{E} & \text { Esfuerzo Plano } \\
\left(1-V^{2}\right) \frac{K_{c}^{2}}{E} & \text { DeformaciónPlana }
\end{array}\right.
$$

donde $\mathrm{E}$ es el módulo de Young, $\mathrm{K}_{\mathrm{c}}$ es la tenacidad de la fractura y $v$ es la razón de Poisson.

El tercer criterio establece que la propagación de la grieta ocurre en la dirección de la mínima densidad de energía de deformación. Para el caso en que sólo el modo I de carga se encuentre presente, el valor crítico de la mínima densidad de energía de deformación $\left(S_{c}\right)$ está dado por Unger (2001):

$$
S_{c}=\frac{2\left(\frac{K_{1}}{\sqrt{2 \pi}}-1\right)}{16 \mathrm{Gz}} K_{c}^{2}
$$

donde $K_{1}$ es el factor de intensidad de esfuerzos para el modo I y Gz es el módulo de corte. 
Para establecer qué teoría de la mecánica de fractura se debe utilizar, es necesario encontrar el diámetro de la zona plástica $\left(r_{p}\right)$ que para el modo de carga I (Anderson, 1995), está definido por:

$$
r_{p}(\theta)=\frac{1}{4 \pi}\left(\frac{K_{1}}{\sigma_{y}}\right)^{2}\left\lceil(1-2 v)^{2}(1+\cos \theta)+\frac{3}{2} \sin ^{2} \theta\right]
$$

donde $\sigma_{\mathrm{y}}$ es el esfuerzo de fluencia y $\theta$ es el ángulo de orientación.

Para que la teoría de la mecánica de la fractura linealmente elástica sea válida, es necesario que la razón del diámetro de la zona plástica y la profundidad de la grieta (a) sea menor a 0.02. Cuando no se cumple esta condición se debe utilizar la teoría de la mecánica de la fractura elastoplástica, la cual incluye otros criterios de falla que describen mejor el estado de los esfuerzos y deformaciones en la punta de la grieta. Estos criterios son: apertura de la grieta (CTOD) y la J-integral.

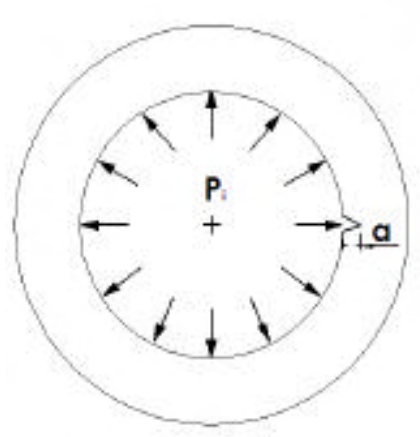

\section{Modelo de prueba}

Con el objetivo de validar el análisis de la fractura del cañón con las simulaciones mecánicas, se realizó el estudio analítico del FIE en un recipiente cilíndrico con una grieta interna longitudinal y se comparó con los resultados obtenidos de las ecuaciones de Perl (Levyet al., 1998).

El recipiente cilíndrico con una grieta interna a lo largo de su longitud y sometido a una presión interna se muestra en la figura 2. Debido a la simetría del recipiente sólo se utilizó la mitad del modelo en la simulación (Figura 3).

Las propiedades mecánicas del acero utilizado para el recipiente cilíndrico fueron las siguientes: un esfuerzo de fluencia de $413.7 \mathrm{MPa}$, módulo de elasticidad de $206.85 \mathrm{MPa}$ y una relación de Poisson de 0.3. En la tabla 1 se muestran los parámetros utilizados para este modelo geométrico.
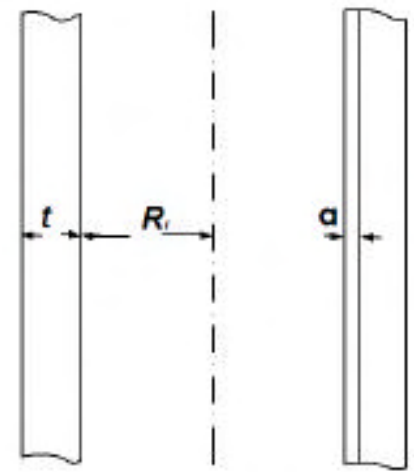

Figura 2. Recipiente cilíndrico a presión interna con grieta interna a lo largo de la longitud

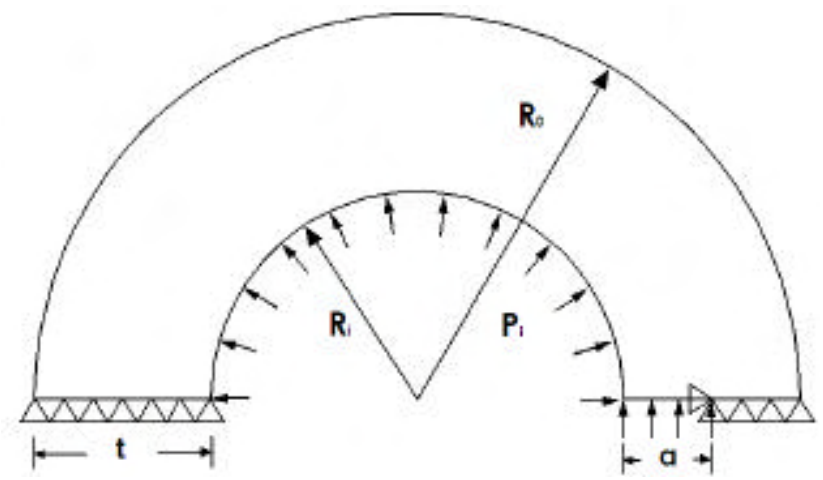

Figura 3. Modelo geométrico en 2-D de un recipiente cilíndrico sometido a presión interna con grieta interna a lo largo de la longitud 
Tabla 1. Parámetros de un recipiente cilíndrico con una grieta interna sometido a una presión interna

\begin{tabular}{cc}
\hline Parámetro & Magnitud \\
\hline Profundidad de la grieta $(\mathrm{a})$ & $2 \mathrm{~mm}$ \\
Espesor del recipiente $(\mathrm{t})$ & $10 \mathrm{~cm}$ \\
Radio exterior $\left(\mathrm{R}_{0}\right)$ & $20 \mathrm{~cm}$ \\
Presión interna $\left(\mathrm{P}_{\mathrm{i}}\right)$ & $10-70 \mathrm{MPa}$ \\
\hline
\end{tabular}

Para el estudio analítico se utilizaron las ecuaciones de Perl:

$$
\begin{aligned}
& \text { la } K=2.66 P_{i}(\pi a)^{1 / 2} K_{\text {IP }} / K_{0} \\
& \text { lb } K=\sigma(\pi a)^{1 / 2} K_{\text {IP }} / K_{0}, \sigma=\frac{2 P_{i} R_{0}^{2}}{R_{0}^{2}-R_{i}^{2}}
\end{aligned}
$$

donde $K$ es el factor de intensidad de esfuerzos, $P_{i}$ es la presión interna, a es la profundidad de la grieta, $K_{\mathrm{p}}$ es el factor de intensidad de esfuerzos, debida a la presión, y $K_{1 p} K_{0}$ se obtiene de la figura 4.
Para la simulación del recipiente se utilizaron 1873 elementos plane82. Se realizó un mallado fino en la región de la grieta con la finalidad de obtener una mejor respuesta en los esfuerzos. Además fue necesario que la punta de la grieta coincidiera con el origen del sistema de coordenadas, que la longitud de los elementos (plane82) cercanos a la grieta fuera aproximadamente un octavo de la profundidad de éste y con la forma de triángulo isósceles alrededor del extremo de la grieta (ANSYS, 2005). Se aplicó presión interna dentro de un rango de $10 \mathrm{MPa}$ a $70 \mathrm{MPa}$, y por simetría se consideró la mitad del recipiente cilíndrico como se muestra en la figura 5.

En la tabla 2, se muestran los resultados del FIE alcanzados con las ecuaciones de Perl, comparados con los obtenidos numéricamente. La diferencia de los resultados alcanzados son menores a un $1 \%$, lo cual es una buena aproximación e indica que la satisfacción de las condiciones en el mallado del modelo geométrico arriba mencionadas producirá resultados aceptables.

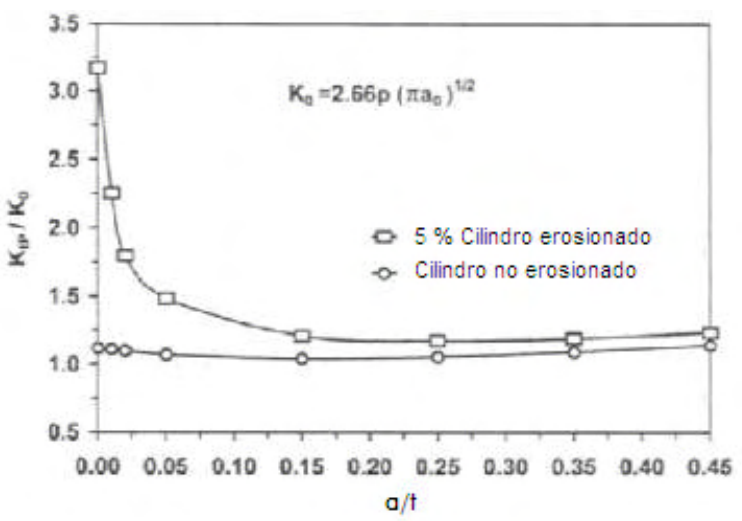

Figura 4. FIEs normalizados, debido a la presión interna en un cilindro agrietado para $5 \%$ de erosión y no erosionado (Levy et al., 1998)

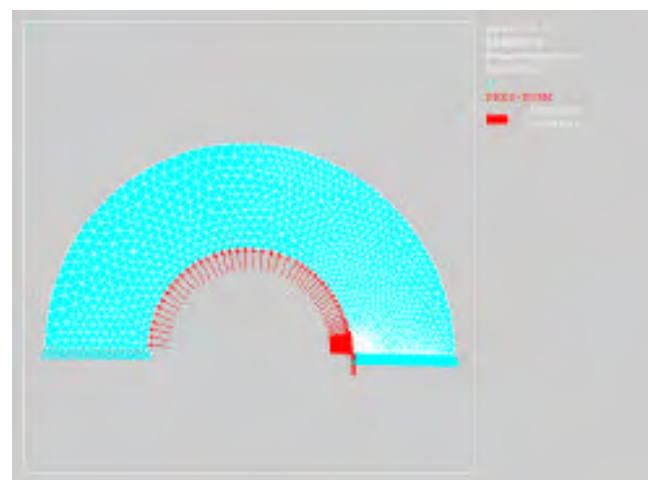

Figura 5. Condiciones de carga y desplazamiento en un recipiente cilíndrico a presión interna. 
DOI: http://dx.doi.org/10.22201/fi.25940732e.2006.07n4.018

A.L. Herrera-May , L.A. Aguilera-Cortés, J. Hernández-Hernández y P.J. García-Ramírez

Tabla 2. FIE en la grieta de $2 \mathrm{~mm}$ de profundidad en un recipiente cilíndrico a una presión interna de $50 \mathrm{M} \mathrm{Pa}$

\begin{tabular}{ccccc}
\hline $\begin{array}{c}\text { Presión Interna } \\
(\mathrm{M} \mathrm{Pa})\end{array}$ & $\begin{array}{c}\text { Ecuación Perl la } \\
\mathrm{MPa}(\mathrm{m}))^{1 / 2}\end{array}$ & $\begin{array}{c}\text { Ecuación Perl lb } \\
\mathrm{MPa}(\mathrm{m})^{1 / 2}\end{array}$ & $\begin{array}{c}\text { Simulación } \\
\mathrm{MPa}(\mathrm{m}))^{1 / 2}\end{array}$ & $\begin{array}{c}\text { Diferencia en \% entre } \\
\text { Simulación y Perl lb }\end{array}$ \\
\hline 10 & 2.2982 & 2.3039 & 2.3185 & 0.634 \\
20 & 4.5965 & 4.6080 & 4.6370 & 0.629 \\
30 & 6.8947 & 6.9119 & 6.9555 & 0.631 \\
40 & 9.1930 & 9.2159 & 9.2740 & 0.630 \\
50 & 11.4912 & 11.5199 & 11.5920 & 0.625 \\
60 & 13.7894 & 13.8239 & 13.9110 & 0.630 \\
70 & 16.0877 & 16.1279 & 16.2290 & 0.627 \\
\hline
\end{tabular}

\section{Análisis del ánima de un cañón en 2-D}

Posteriormente, se realizó un análisis de la distribución de los esfuerzos en el ánima de un cañón de 3"/50 calibre, con un acero ASTM A723 (Koh, 1996), cuyas propiedades se indican en la tabla 3.

Tabla 3. Propiedades mecánicas del acero ASTM A 723 utilizadas en la simulación mecánica

\begin{tabular}{cc}
\hline Módulo de elasticidad & $200 \mathrm{GPa}$ \\
Esfuerzo de fluencia & $1170 \mathrm{MPa}$ \\
Esfuerzo último & $1262 \mathrm{MPa}$ \\
Tenacidad de la fractura & $148.3 \mathrm{MPa}(\mathrm{m})^{1 / 2}$ \\
\hline
\end{tabular}

El equipo utilizado en la simulación fue una laptop con procesador Pentium 4 con $1.60 \mathrm{GHz}$, 480 MB en RAM y 60 GB en disco duro.

Primero se consideró el modelo geométrico en 2-D de un sector del ánima del cañón (Figura 6), aprovechando la simetría del ánima y con la finalidad de optimizar el tiempo de cómputo. En este caso, se despreció el efecto de las grietas internas con la intención de conocer los esfuerzos en los filetes del rayado a una presión interna de $95 \mathrm{MPa}$ y posteriormente, se compararon con los resultados de un segundo caso que incluye grietas internas alrededor del filete. En ambos casos, las dimensiones en el ánima del cañón de 3"/50 calibre (medidos alrededor de la posición de la grieta interna) son las siguientes: radio interno de
$7.62 \mathrm{~cm}$, espesor de $5.66 \mathrm{~cm}$, profundidad del rayado de $1 \mathrm{~mm}$.

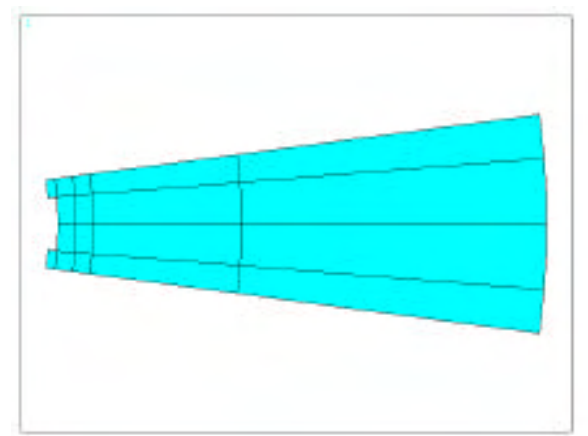

Figura 6 . Modelo geométrico en $2-D$ del ánima de un cañón 3"/50 calibre

Se utilizó un mallado con 1713 elementos plane82 y 5222 nodos. Alrededor del filete del rayado se realizó un mallado más fino con elementos triangulares que formaron una trayectoria semicircular (Figura 7). Se aplicó una presión interna de $95 \mathrm{MPa}$ y se consideraron restricciones de simetría en la parte superior e inferior del modelo geométrico.

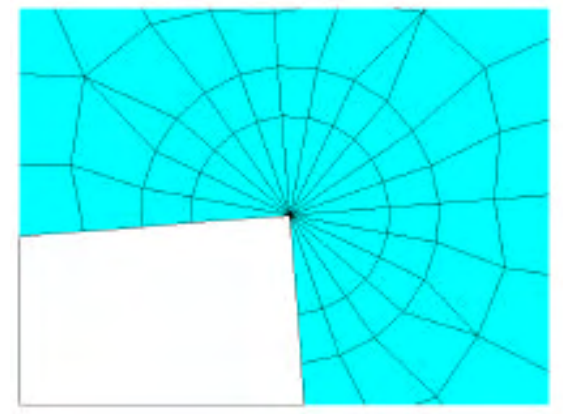

Figura 7. Mallado fino alrededor de un filete del rayado del ánima de un cañón 3"/50 calibre 
La distribución de los esfuerzos (Von Mises) obtenidos en este sector del ánima del cañón se indican en la figura 8. En ella, se observa que existe una gran concentración de esfuerzos en las cercanías de los filetes (Figura 9), alcanzando un esfuerzo máximo de $496 \mathrm{MPa}$. Aunque es un valor muy grande, no supera el esfuerzo de fluencia del material, por lo que bajo esta condición de carga y sin considerar grietas internas no producirá falla en el material.
En un segundo análisis del sector del ánima, se consideraron dos grietas internas de $0.4 \mathrm{~mm}$ de profundidad cerca del rayado con la misma condición de presión (95 $\mathrm{MPa}$ ) del caso anterior. En este caso, se utilizó un mallado de 1889 elementos con 5740 nodos, obteniendo una densidad de malla fina en la cercanía de las grietas como se muestra en la figura 10 , en donde además se visualizan los vectores de presión aplicados en la cara transversal y so bre la grieta superior.

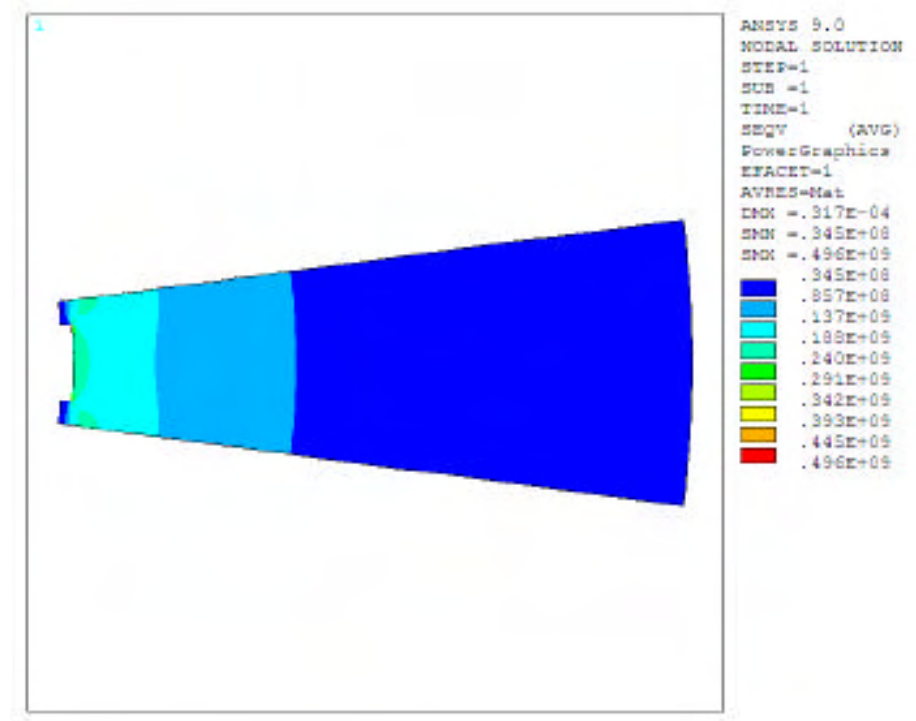

Figura 8. Distribución de los esfuerzos de Von Mises ( $\mathrm{Pa}$ ) en un sector del ánima de un cañón de 3"/50 calibre sin grietas

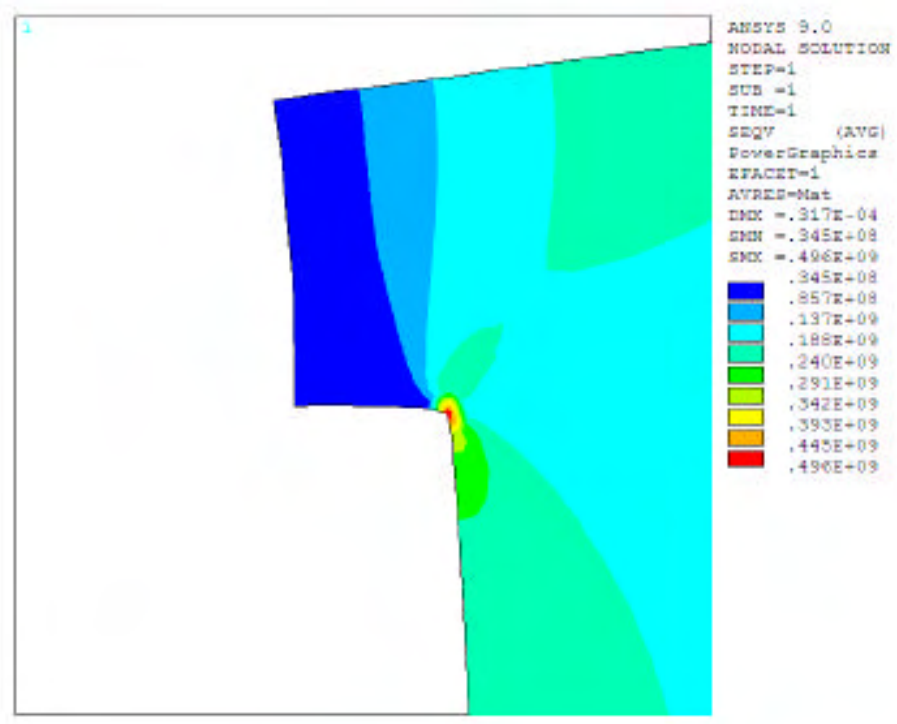

Figura 9. Esfuerzos de Von $\mathrm{M}$ ises ( $\mathrm{Pa}$ ) en el filete supe rior en un sector del ánima de un cañón de 3"/50 calibre sin grietas 


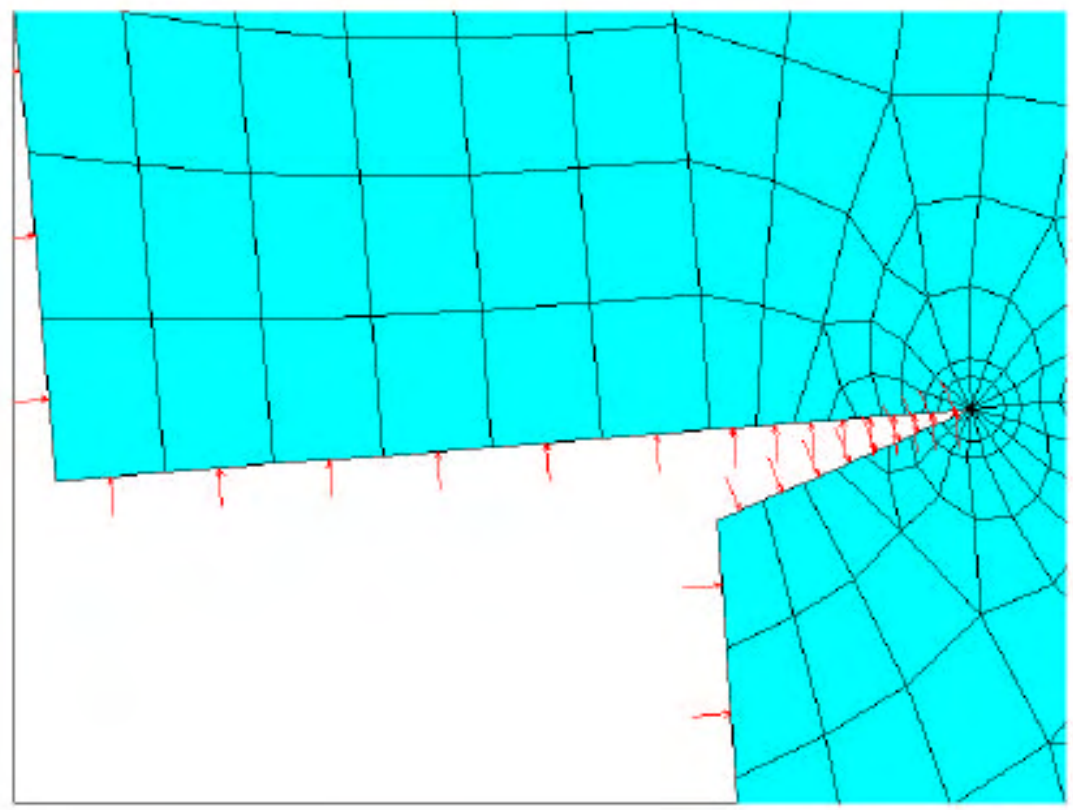

Figura 10. M allado alrededor de la grieta interna supe rior en un sector del ánima del cañón 3"/50 calibre

Los resultados obtenidos en la distribución de los esfuerzos (Figura 11) indicaron un valor máximo de $835 \mathrm{MPa}$ en las puntas de las dos grietas (nodos 3769 y 1165). En comparación con el sector del ánima sin grieta, se obtiene un incremento del $68.35 \%$ en los esfuerzos de Von Mises. A pesar de ello, el esfuerzo máximo aún no supera el esfuerzo de fluencia del material. En las figuras $12-13$ se observó que la distribución de los esfuerzos alrededor de las grietas tiene la forma de ala de mariposa, la cual es común para este tipo de grieta con dicha condición de carga (modo I).

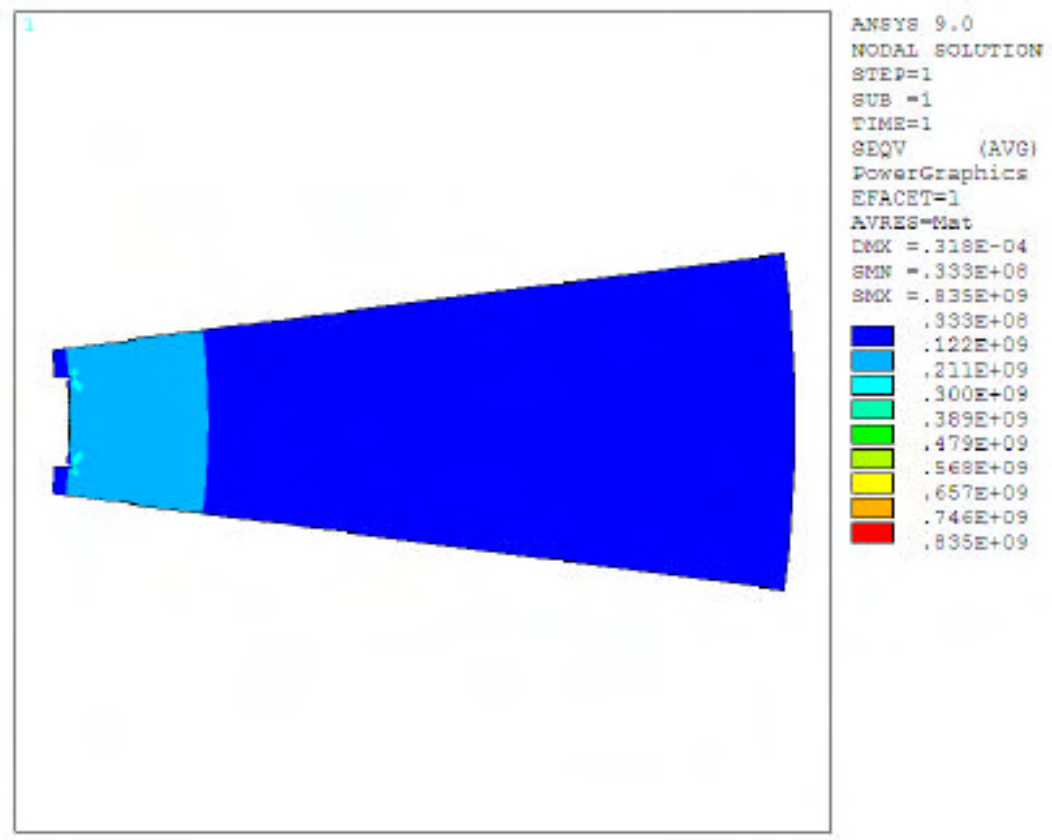

Figura 11. Distribución de los esfuerzos de Von M ises ( $\mathrm{Pa}$ ) en un sector del ánima de un cañón $3 " / 50$ calibre con dos grietas internas de $0.4 \mathrm{~mm}$ de profundidad 


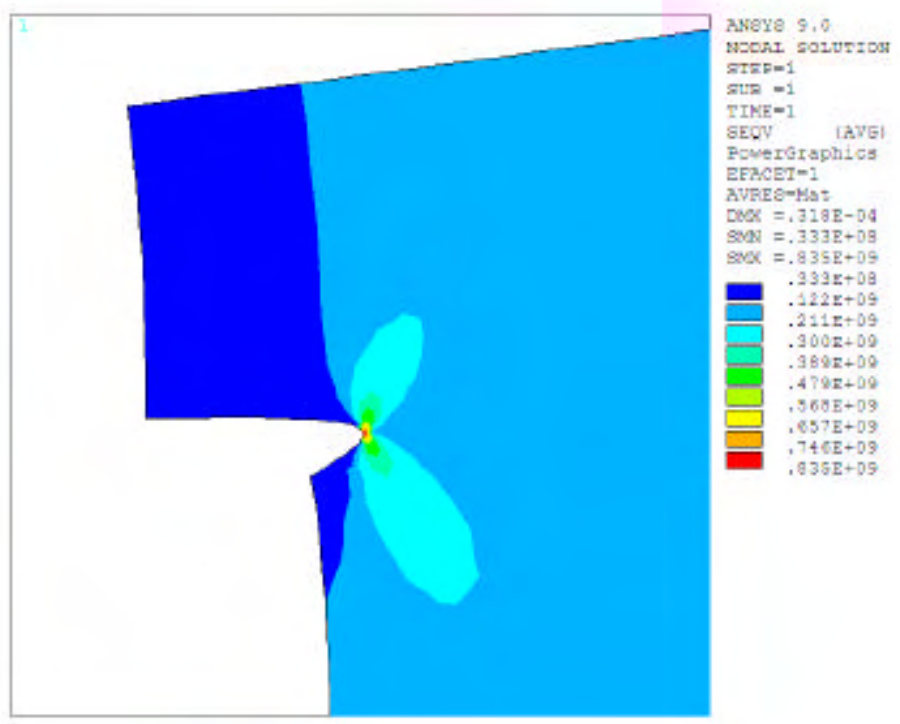

Figura 12. Esfuerzos de Von $M$ ises $(\mathrm{Pa})$ en la grieta supe rior de $0.4 \mathrm{~mm}$ en un sector del ánima de un cañón de $3 " / 50$ calibre

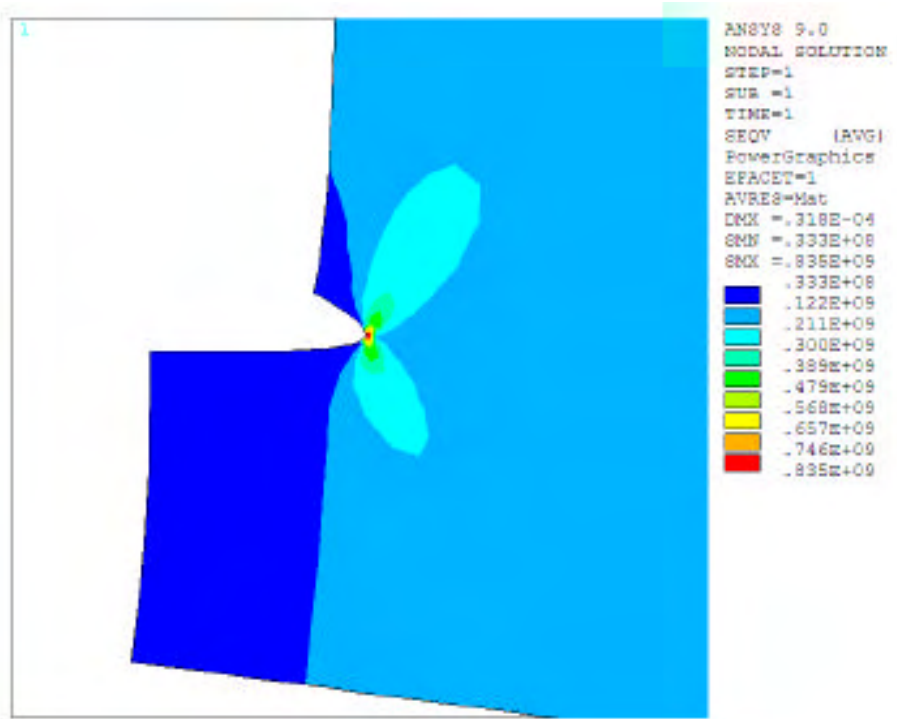

Figura 13. Esfuerzos de Von $M$ ises $(\mathrm{Pa})$ en la grieta superior de $0.4 \mathrm{~mm}$ en sector del ánima de un cañón de 3"/50 calibre

Para encontrar el FIE en la punta de la grieta superior, se consideró una trayectoria de nodos perfectamente alineados en dirección del eje " $x$ ", con lo cual se obtuvo un valor de $9.534 \mathrm{MPa}(\mathrm{m})^{1 / 2}$ para el modo I.

Con la finalidad de poder establecer la validez de la teoría de la fractura linealmente elástica, se obtuvo el diámetro de la zona plástica (Figura 14) en la punta de la grieta, utilizando la ecuación 3.
Su diámetro máximo para este caso fue de 0.0075 $\mathrm{mm}$, con lo cual se encontró una razón $r_{p} / a=0.019$, lo que garantiza la validez de la teoría de la fractura linealmente elástica.

Por la simetría en la forma de las dos grietas y con la influencia de la misma condición de carga, se observó que ambas mantienen la misma magnitud de los esfuerzos y FIE en sus puntas. 


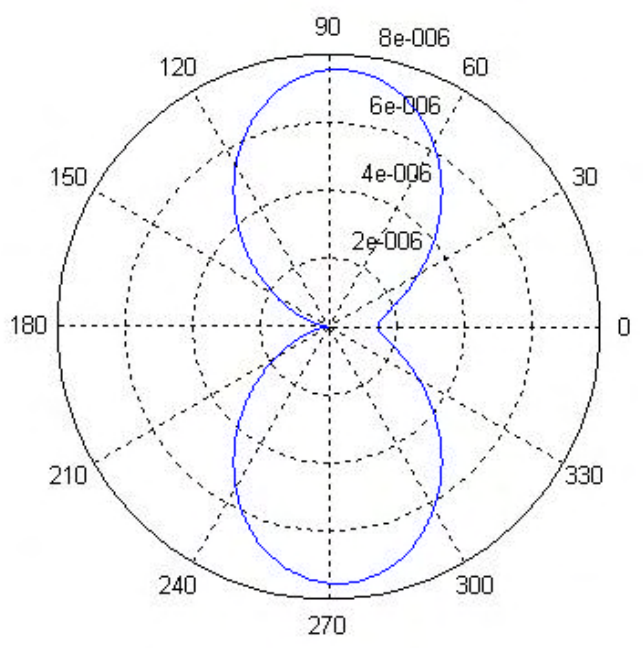

Fig. 14. Región plástica en la punta de la grieta supe rior de $0.4 \mathrm{~mm}$ de profundidad en un sector del ánima de un cañón $3 " / 50$ calibre, modelo en 2D

Con base a la magnitud del FIE obtenido en la grieta interna superior, se encontraron los valores de la razón de energía liberada $G$ y la mínima densidad de energía de deformación S, así como los valores críticos para que la grieta empiece a propagarse (Tabla 4). Los resultados obtenidos indican que el ánima del cañón puede seguir operando bajo condiciones estables para esta condición de carga.

Tabla 4. Criterios de falla para el modo I de carga en la grieta interna de $0.4 \mathrm{~mm}$ del ánima de un cañón de $3 " / 50$ calibre

\begin{tabular}{clc} 
Razón de Energía Liberada $(\mathrm{G})$ & $\left(\mathrm{G}_{\mathrm{I}}\right)$ & $413.58 \mathrm{~J} / \mathrm{m}^{2}$ \\
& $\left(\mathrm{G}_{\mathrm{IC}}\right)$ & $100,067.65 \mathrm{~J} / \mathrm{m}^{2}$ \\
$\begin{array}{c}\text { Mínima Densidad de } \\
\left(\mathrm{S}_{\mathrm{I}}\right)\end{array}$ & $118.17 \mathrm{~J} / \mathrm{m}^{2}$ \\
Energía de Deformación $(\mathrm{S})$ & $\left(\mathrm{S}_{\mathrm{IC}}\right)$ & $28,590.76 \mathrm{~J} / \mathrm{m}^{2}$ \\
\hline
\end{tabular}

Los modelos geométricos anteriores en 2-D son muy útiles para conocer la distribución de los esfuerzos y el FIE en la sección más crítica del ánima de un cañón (inicio del rayado). Además, tienen la ventaja que no se necesita un mallado demasiado grande para sus análisis, pero está limitado a grietas simétricas y longitudinales. Para el caso de grietas que no cumplan con este requisito, se necesita un análisis en un modelo geométrico en 3-D.

\section{Análisis del ánima de un cañón en 3-D}

Con el objetivo de considerar el efecto que producen las grietas semicirculares en el ánima del cañón de 3"/50 calibre, se realizó un modelo en 3-D con los mismos radios, profundidad de rayado y material del caso en 2-D. La grieta tiene una forma semicircular de $0.4 \mathrm{~mm}$ de radio, la cual está localizada a la mitad de los dos extremos del rayado. El mallado (Figura 15) del sector del ánima, está compuesto de 19003 elementos del tipo solid95 con 30276 nodos. Este mallado es muy denso porque alrededor de la grieta se realizó un mallado muy fino (Figura 16), para obtener una forma semicircular alrededor de ésta y con elementos triangulares muy pequeños alrededor de ella, que garanticen una solución confiable.

Como condición de carga se aplicó una presión interna de $95 \mathrm{MPa}$ (Figura 17), con lo cual se obtuvo una distribución de los esfuerzos (Von Mises) del sector como se muestra en la figura 18. Alrededor de los filetes del rayado se encontraron magnitudes de esfuerzos cercanos a $484 \mathrm{MPa}$ como se observa en la figura 19. Los esfuerzos máximos se encuentran localizados alrededor de la grieta semicircular (Figura 20), siendo $865 \mathrm{MPa}$ el valor máximo y ubicado en el extremo frontal de la grieta (nodo 25392). Este esfuerzo no supera el límite de fluencia del material, por lo que no fallará por fluencia para esta condición de carga. 
DOI: http://dx.doi.org/10.22201/fi.25940732e.2006.07n4.018

Análisis de los esfuerzos y fractura en el ánima de un cañón de 3"/ 50 calibre

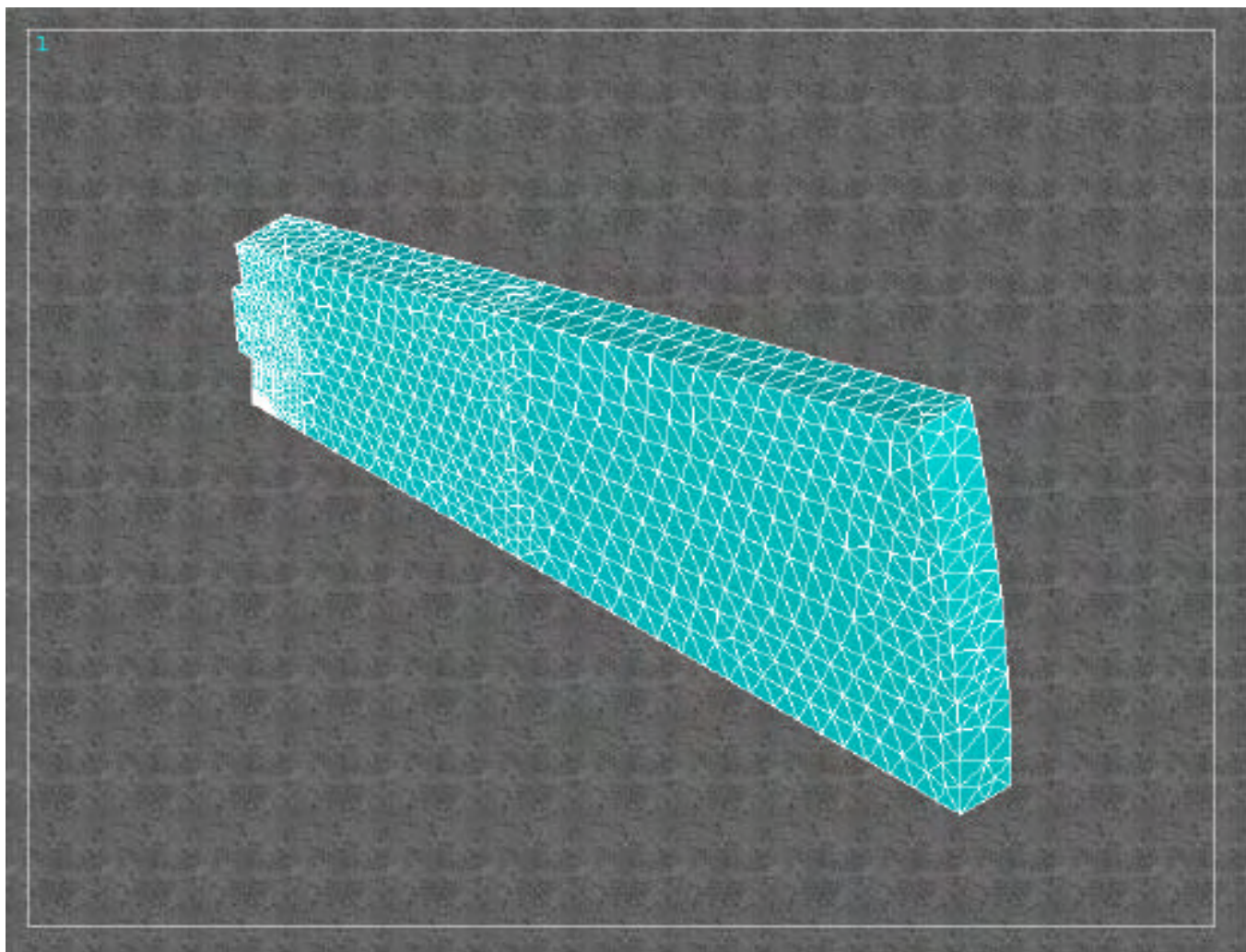

Figura 15. Mallado del sector del ánima en 3-D, con grieta semi cir cular de $0.4 \mathrm{~mm}$ de radio, de un cañón de $3 " / 50$ calibre

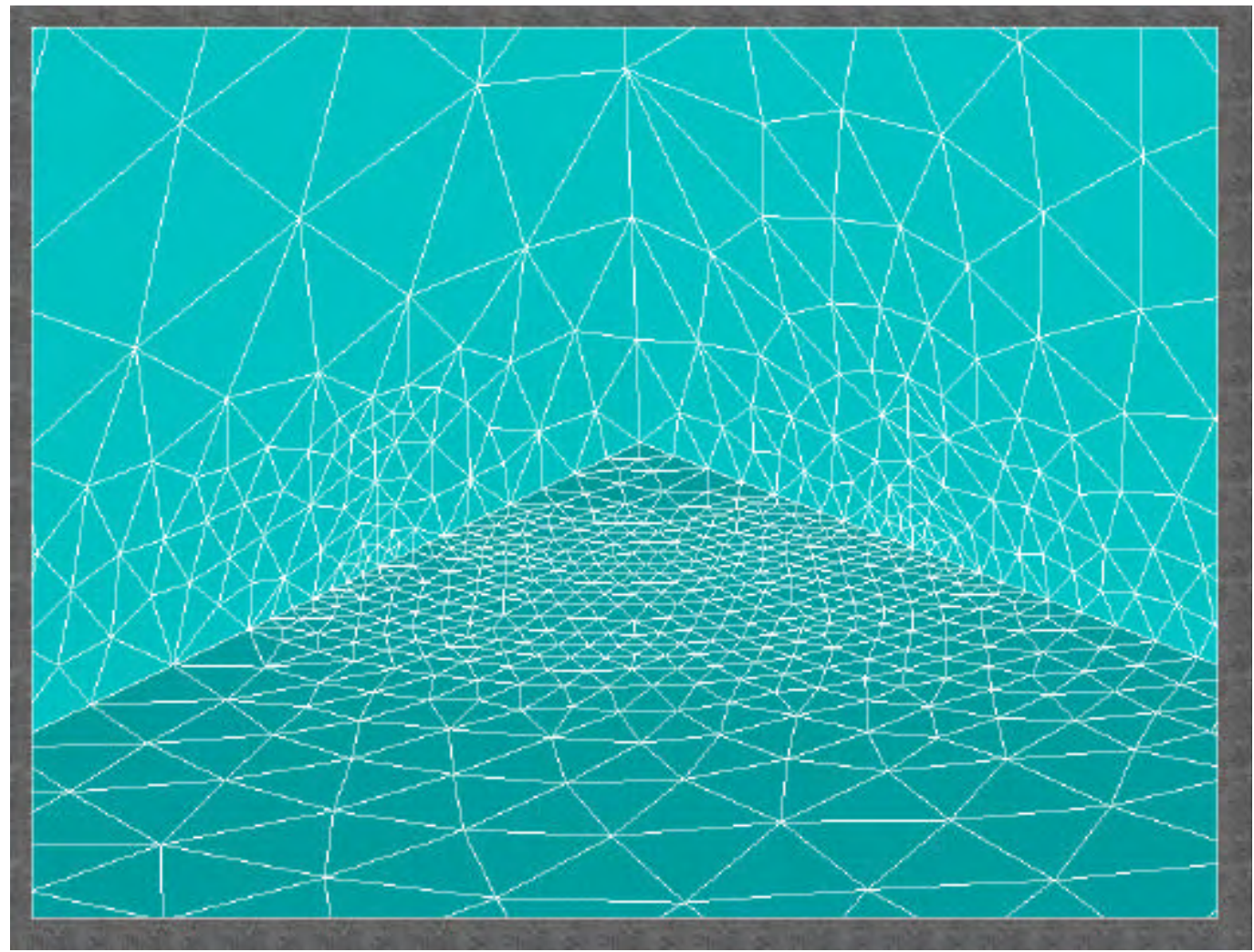

Figura 16. M allado fino alrededor de la grieta semi cir cular del sector del ánima en 3-D de un cañón de 3"/50 calibre 


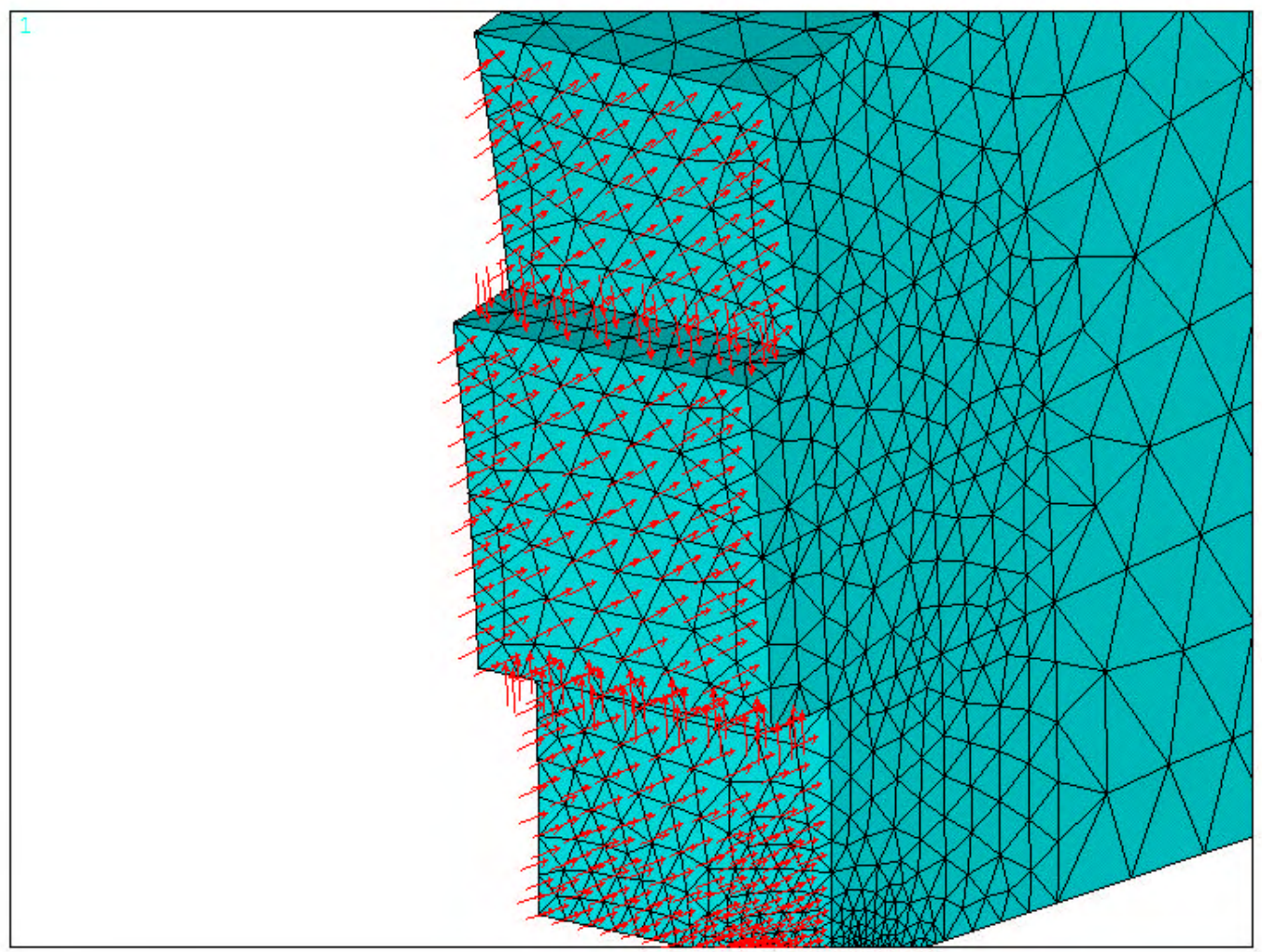

Figura 17. Presión interna de $95 \mathrm{MPa}$ en el sector del ánima en 3-D de un cañón de 3"/50 calibre

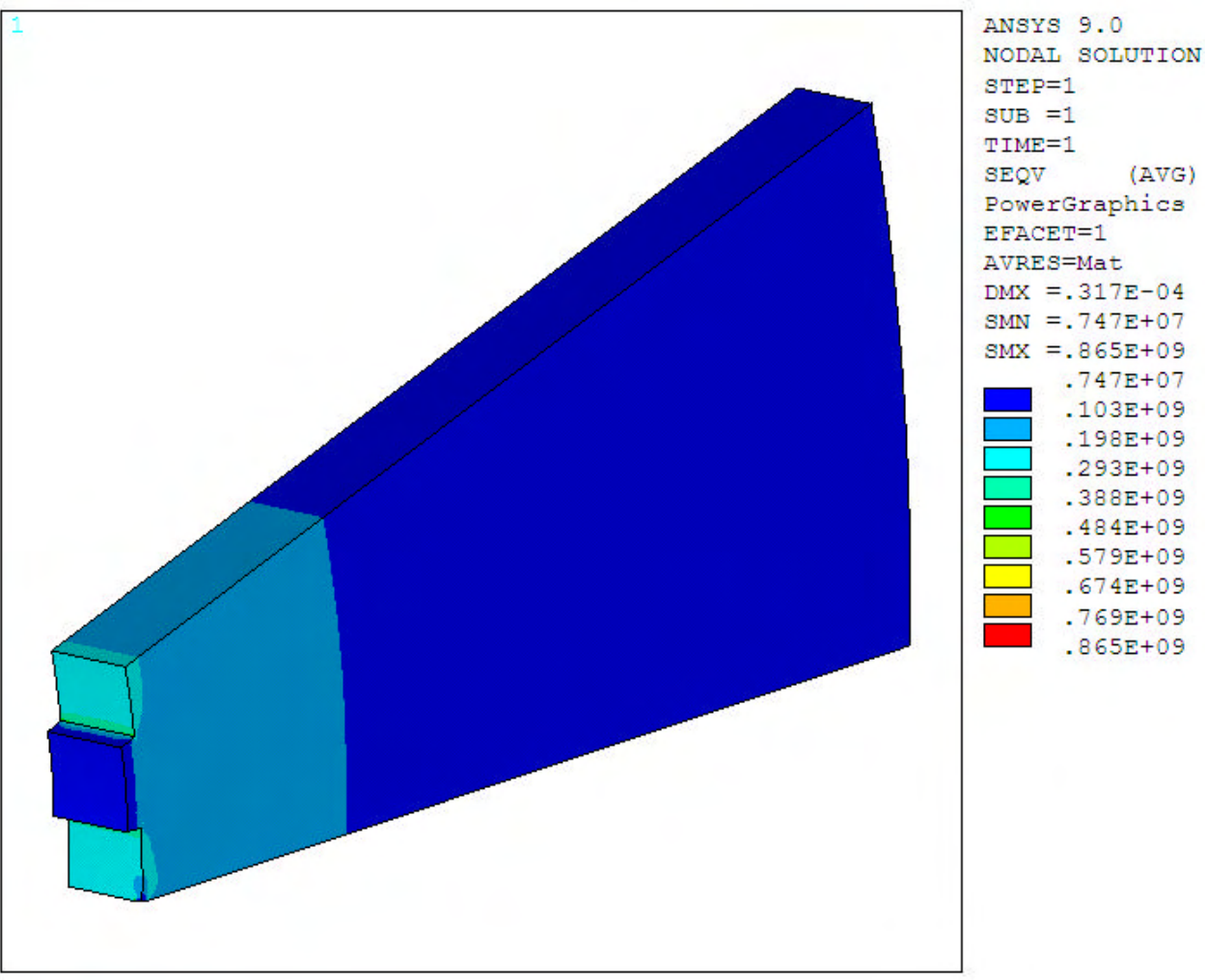

Figura 18. Esfuerzos de Von Mises ( $\mathrm{Pa}$ ) en el sector del ánima en 3-D de un cañón 3"/50 calibre 


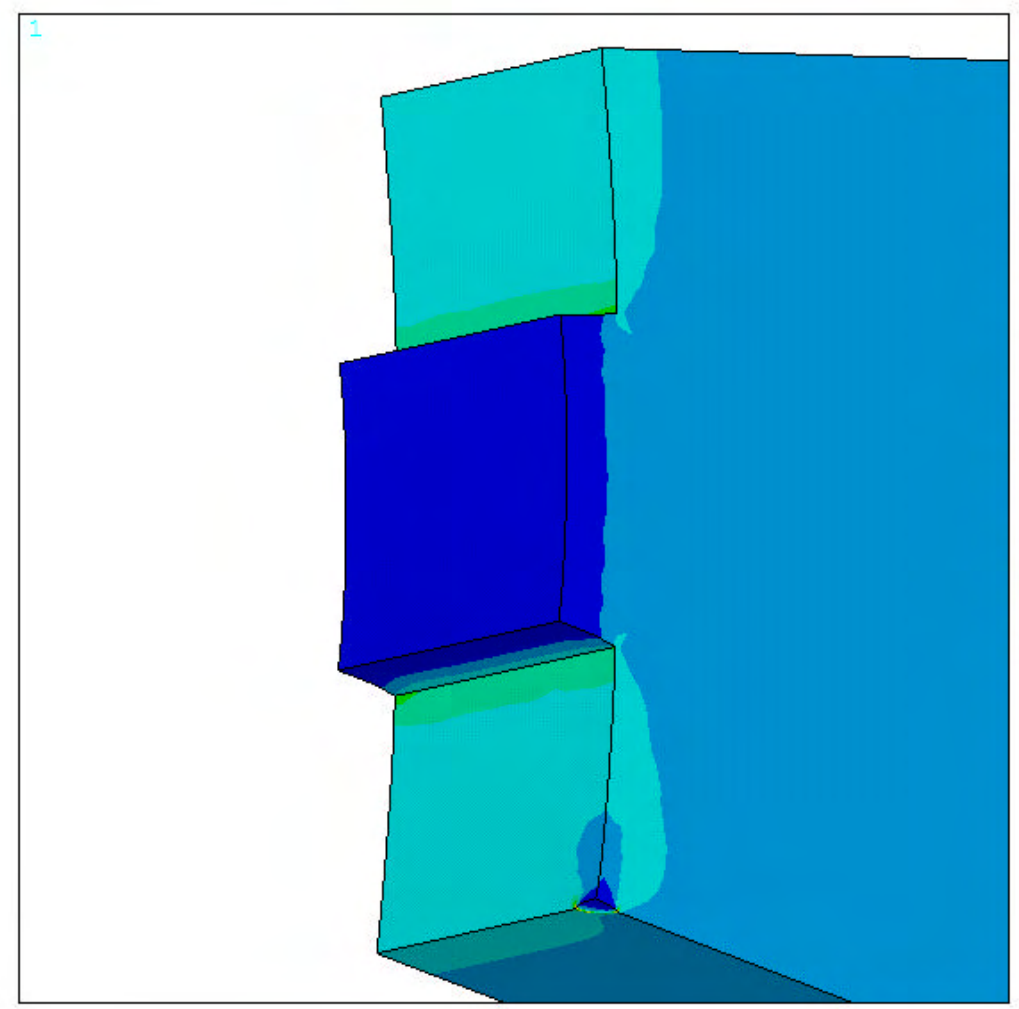

ANSYS 9.0

NODAI SOLUTION

$S T E P=1$

SUB $=1$

TIME $=1$

SEQV

PowerGraphics

EFACET $=1$

AVRES=Mat

$D M X=.317 \mathrm{E}-04$

SMN $=.747 \mathrm{E}+07$

$\operatorname{SMX}=.865 \mathrm{E}+09$

$.747 \mathrm{E}+07$

$.103 \mathrm{E}+09$

$.198 \mathrm{E}+09$

$.293 E+09$

$388 \mathrm{E}+09$

$.484 \mathrm{E}+09$

$.579 \mathrm{E}+09$

$.674 \mathrm{E}+09$

$.769 \mathrm{E}+09$

$.865 \mathrm{E}+09$

Figura 19. Esfuerzos de Von M ises (Pa) en la parte interna del sector del ánima en 3-D de un cañón 3"/50 calibre

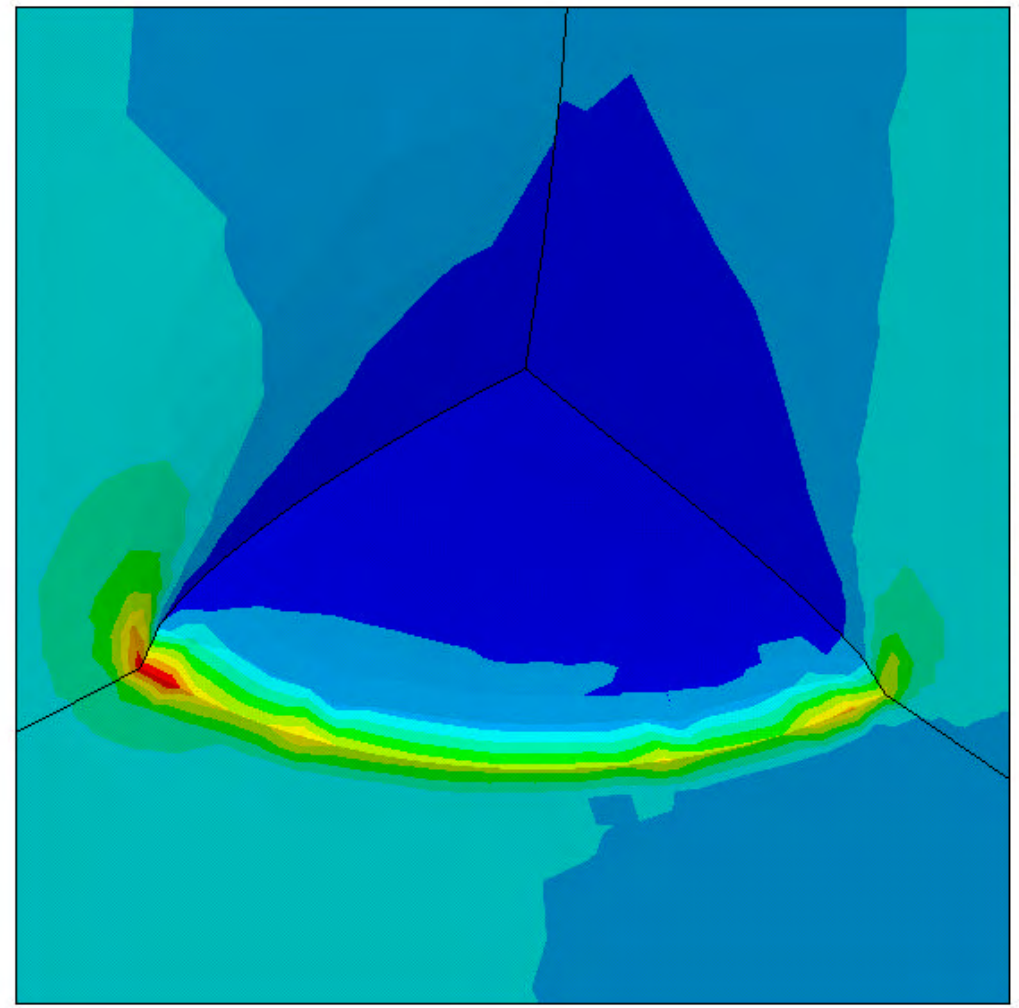

ANSYS 9.0

NODAI SOLUTION

STEP $=1$

SUB $=1$

TIME $=1$

$S E Q V$

(AVG)

PowerGraphics

EFACET $=1$

AVRES=Mat

$\mathrm{DMX}=.317 \mathrm{E}-04$

SMN $=.747 \mathrm{E}+07$

SMX $=.865 \mathrm{E}+09$

$.747 \mathrm{E}+07$

$.103 E+09$

$.198 \mathrm{E}+09$

$.293 E+09$

$.388 \mathrm{E}+09$

$.484 \mathrm{E}+09$

$.579 E+09$

$.674 \mathrm{E}+09$

$.769 \mathrm{E}+09$

$.865 \mathrm{E}+09$

Figura 20. Esfuerzos de Von M ises (Pa) alrededor de la grieta semi cir cular del sector del ánima de un cañón de 3"/50 calibre 
Para el análisis de la fractura se obtuvo el FIE en los dos extremos de la grieta. Para el extremo frontal de la grieta se logró un valor de 5.203 $\mathrm{MPa}(\mathrm{m})^{1 / 2}$ y para el extremo lateral se encontró una magnitud de $4.782 \mathrm{MPa}(\mathrm{m})^{1 / 2}$. Con la finalidad de poder establecer la validez de la teoría de fractura linealmente elástica, de acuerdo a la ecuación 3, se encontró la forma y tamaño de la zona plástica en los dos extremos de de la grieta (Figuras 21-22). De la gráfica anterior, se observó que el diámetro máximo de la zona plástica en el extremo frontal es aproximadamente $0.0023 \mathrm{~mm}$, obteniendo una razón $r_{p} / a=0.00575$ y en el extremo lateral su diámetro máximo es de $0.0019 \mathrm{~mm}$ produciendo una razón $r_{0} / a=0.00475$. Estas razones son menores de 0.02 , y por ende, los criterios de falla de la teoría de la fractura linealmente elástica son válidos. Los criterios de falla son mostrados en la tabla 5 y se observa que la grieta no provocará falla por fractura para este tipo de grieta y condiciones de carga, ya que estos criterios se encuentran lejos de su valor crítico.

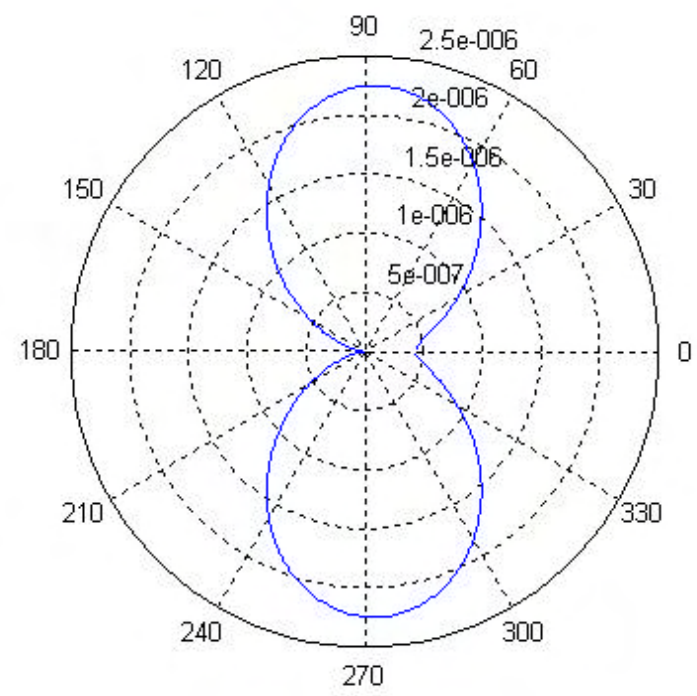

Figura 21. Región plástica en el extremo frontal de la grieta semi cir cular de $0.4 \mathrm{~mm}$ de radio de un cañón de 3"/50 calibre, modelo en 3D

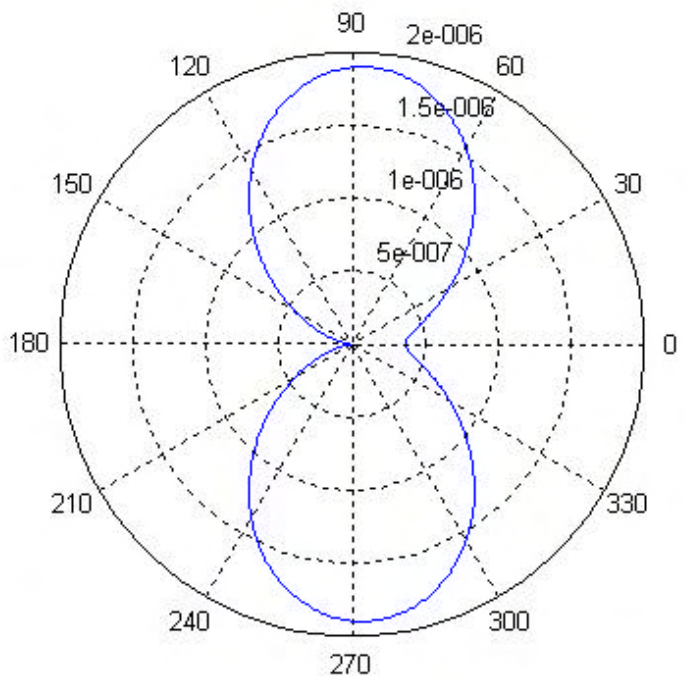

Figura 22. Región plástica en el extremo lateral de la grieta semi cir cular de $0.4 \mathrm{~mm}$ de radio de un cañón de 3"/50 calibre, modelo en 3D 
DOI: http://dx.doi.org/10.22201/fi.25940732e.2006.07n4.018

Análisis de los esfuerzos y fractura en el ánima de un cañón de 3"/ 50 calibre

Tabla 5. Criterios de falla para el modo I de carga en la grieta semi cir cular interna de $0.4 \mathrm{~mm}$ del ánima de un cañón de $3 " / 50$ calibre

Razón de Energía Liberada

(G) en $\mathrm{J} / \mathrm{m}^{2}$
Mínima Densidad de Energía de Deformación

(S) en $\mathrm{J} / \mathrm{m}^{2}$
$\left(G_{1}\right)$

E.F.1

E.L. ${ }^{2}$
$\left(G_{1}\right)$

$100,067.65$

$100,067.65$
$\left(S_{1}\right)$

35.19

29.73
$\left(\mathrm{S}_{\mathrm{IC}}\right)$

$28,590.76$
1 Extremo frontal

2 Extremo lateral

\section{Conclusiones}

El método del elemento finito por medio del paquete comercial ANSYS9.0 ${ }^{\circ}$ fue una herramienta muy útil en el análisis de los esfuerzos del ánima de un cañón de 3"/50 calibre. En los casos mostrados del ánima, los esfuerzos máximos de Von Mises no superaron el esfuerzo de fluencia del acero ASTM A723, por lo que es segura la operación del cañón bajo la condición de presión interna de $95 \mathrm{MPa}$ para los tipos de grietas analizados. En el análisis se observó una concentración mayor de los esfuerzos en las puntas y extremos de las grietas, el cuál resulta lógico. La generación de las grietas internas de $0.4 \mathrm{~mm}$ de longitud en el modelo geométrico en 2-D, provocó un incremento del $68.35 \%$ en los esfuerzos de Von Mises con una presión interna de $95 \mathrm{MPa}$. El modelo geométrico en 2-D es muy útil cuando se tienen grietas longitudinales en el ánima, ya que el mallado no es tan extenso y el tiempo de cómputo es relativamente menor.

Para incluir el efecto de una grieta semicircular se realizó un modelo geométrico en 3-D del sector del ánima del cañón, en donde se obtuvo un mallado muy fino alrededor de la grieta para obtener resultados confiables. Los esfuerzos máximos de Von Mises obtenidos en este modelo se concentran en la punta de la grieta, pero sin superar los esfuerzos de fluencia del material.

En el análisis de fractura se consideraron los criterios de falla de la mecánica de la fractura linealmente elástica (LEFM). En todos los casos, se demostró que se podían aplicar estos criterios, ya que no se superó la razón $r_{p} / a=0.02$. Para el sector del ánima con una grieta de $0.4 \mathrm{~mm}$, se visualizó que el factor de intensidad de esfuerzos está lejano del valor crítico, lo cual garantiza una operación segura del ánima bajo una presión interna de $95 \mathrm{MPa}$. En el análisis de 3-D, se consideró una grieta semicircular de $0.4 \mathrm{~mm}$ de radio, sujeta a la misma condición de carga. Bajo esta condición fue posible aplicar los criterios de falla de la LEFM, los cuáles demostraron que se encuentra lejana una posible falla por fractura para esta condición de carga. La utilización de los modelos geométricos propuestos en 2-D y 3-D del ánima de un cañón con ANSYS9.0๑, reduce el tiempo de cómputo y son de fácil manejo para la obtención de los esfuerzos y el FIE que le ocasionan grietas internas. La simulación mecánica de un modelo de prueba de un recipiente cilíndrico fue realizada para validar sus resultados computacionales con las ecuaciones de Perl, obteniendo un porcentaje de error menor al $1 \%$.

\section{Referencias}

Anderson T.L. (1995). Fracture Mechanics Fundamentals and Appli ca tions. Ed. CRC.

ANSYS9.0, M anual de ANSYS, 2005.

Bache J.F. (1996). Armas navales I. Heroica Escuela Naval Militar, México, DF.

Galavíz J.D. (2003). Análisis y propuesta de solución a la grieta generada en el gancho de una grúa viajera. Tesis de maestría, FIMEE, Universidad de Gto. México.

González J.L. (1998). M ecánica de fractura. Ed. Limusa, México, DF.

Koh S.K.(1996). Fatigue Life Simulation and Estimation of an Autofrettaged ThickWalled Pressure Vessel with an External Groove. Int. Journal of Fatigue, Vol. 18, No. 1, pp. 49-56.

Levy C., Perl M. and Fang H. (1998). Cracks Emanating From an Erosion in a Pres sur ized 
DOI: http://dx.doi.org/10.22201/fi.25940732e.2006.07n4.018

A.L. Herrera-May , L.A. Aguilera-Cortés, J. Hernández-Hernández y P.J. García-Ramírez

Autofrettaged Thick-Walled Cylinder-Part I: Semi-Circular and Arc Erosions. Journal of PressureVessel Tech nology, Vol. 120, pp. 349-353.

Levy C., Perl M. and Ma Q. (2003). The Influence of Finite Three-Dimensional Multiple Axial Erosions on the Fatigue Life of Partially Autofrettaged Pressurized Cylinders. Journal of Pressure Vessel Technology, Vol. 125, pp. 379-384.

Underwood J.H. and Andino M.J. (1998). Effects of Initial Cracks and Firing Envi- ronment on Cannon Fatigue Life. Fatigue Design. Tech nical Research Center of Fin- land, Espoo, Finland, Vol. 2, pp. 491-500.

Under wood J.H., Parker A.P., Troiano E, Vigi lante G.N. and Witherell M.D. (2001). Fatigue and Hydrogen Cracking in Cracking in Cannons with Mechan ical and Thermal Residual Stress. Advances in Fracture Research, Proceed ings of ICF10, Pergamon.

Unger J.D. (2001). Analytical Fracture Mechanics. 1ra edición, Dover Publi ca tions.

\section{Semblanza de los autores}

A gustín Leobardo H errera-M ay . Ingeniero mecánico electricista egresado de la Universidad Veracruzana en el año 2000. Obtuvo el grado de maestro en ingeniería mecánica en 2002 por la FIMEE de la Universidad de Guanajuato. Sus áreas de interés son diseño mecánico, vibraciones mecánicas, elemento finito, fractura, MEMS y soft ware de CAD. En el 2002, trabajó como profesor de la FIMEE de la Universidad de Guanajuato y de 2003 a 2005 como profesor de tiempo completo en la Escuela de Ingenieros de la Armada de México. Actualmente es investigador del Centro de Investigación en Micro y Nanotecnología de la Universidad Veracruzana. Asimismo este año recibió el reconocimiento de perfil deseable de PROME P.

Luz Antonio Aguilera-Cortés. Obtuvo la licenciatura, maestría y doctorado en ingeniería mecánica en la Universidad de Guanajuato en 1988, 1990 y 1995, respectivamente. Ha realizado investigaciones en el estudio de sistemas dinámicos incluyendo modelación, simulación y análisis, con enfoque hacia las vibraciones mecánicas de cuerpos flexibles. Tiene publicados 4 artículos en revistas internacionales, 7 en revistas nacionales y 40 artículos en foros con arbitraje a nivel nacional e internacional. De 1992 a 1996, fue miembro del Sistema Nacional de Investigadores (SNI) de donde en el 2005 recibió el SNI nivel 1. Actualmente está adscrito a la Facultad de Ingeniería Mecánica, Eléctrica y Electrónica (FIMEE) de la Universidad de Guanajuato con nombramiento de Profesor Asociado C.

José Hernández-Hernández. Ingeniero en ciencias navales egresado de la Heróica Escuela Naval Militar en 1991, obtuvo la licenciatura en ingeniería naval de la Universidad Veracruzana en 2002, y actualmente es estudiante de la maestría de ingeniería mecánica del Instituto de Ingeniería de la Universidad Veracruzana. Ha ocupado diferentes cargos dentro de la Secretaría de Marina en buques de la Armada, siendo su actual cargo el dejefe de la carrera deingeniero mecánico naval de la Escuela de Ingenieros de la Armada de México.

Pedro Javier García-Ramírez. Ingeniero indus trial en electrónica egresado del ITV en 1993. Obtuvo el grado de maestría en ciencias de la electrónica en 1997 y el doctorado en ciencias de la electrónica en 2000 en el INAOE. Desde e I año 2001, es profesor de la Facultad de Ingeniería de la Universidad Veracruzana. Es miembro del Sistema Nacional de Investigadores (SNI) desde el 2002 y cuenta con perfil PROMEP a partir del 2004. Asimismo, es miembro de la IEEE desde el 2004. Actualmente es director del Centro de Investigación en Micro y Nanotecnología de la Universidad Veracruzana. 